\title{
EXTRAÇÃO DE POLISSACARÍDEOS DE BIOMASSA MICELIAL DE Pleurotus sajor-caju
}

\author{
K. L. HEINZ ${ }^{1}$, M. L. L. SILVEIRA ${ }^{2}$, M. BONATTI-CHAVES ${ }^{2}$, F. R. SMIDERLE ${ }^{3}$, M. IACOMINI ${ }^{3}$, \\ S. A. FURLAN ${ }^{2}$ \\ ${ }^{1}$ Universidade da Região de Joinville, Departamento de Engenharia Química, IC/CNPq \\ ${ }^{2}$ Universidade da Região de Joinville, Departamento de Engenharia Química \\ ${ }^{3}$ Universidade Federal do Paraná, Departamento de Bioquímica e Biologia Molecular \\ E-mail para contato: karyn_lanna@hotmail.com
}

\begin{abstract}
RESUMO - Os fungos despertam interesse devido seu valor gastronômico, sendo considerados nutricionalmente completos, e devido a possibilidade de extração de substâncias bioativas. Entre estas estão polissacarídeos da parede celular destes fungos, que apresentam atividades biológicas. $\mathrm{O}$ objetivo do trabalho foi extrair os polissacarídeos presentes na biomassa micelial, obtida de cultivo submerso de Pleurotus sajor-caju. Após a produção de biomassa por cultivo submerso, foi realizada a extração aquosa a frio e a quente dos polissacarídeos. Estes extratos foram fracionados e purificados por diferentes métodos e analisados quanto composição monossacarídica e homogeneidade. Os resultados obtidos demonstram que o extrato aquoso frio possui frações formadas por unidades de galactose e manose e o extrato aquoso quente apresentou frações formadas por unidades de manose e glucose.
\end{abstract}

\section{INTRODUÇÃO}

Os cogumelos do gênero Pleurotus possuem elevado teor de proteínas com a presença de aminoácidos essenciais, fibras, carboidratos, baixo teor de gorduras, presença de vitaminas do complexo B e de minerais (Manzi e Pizzoferato, 2000; Bonatti et al., 2004; Rampinelli et al., 2010).

Entre os carboidratos estão os $\beta$-glucanos, polissacarídeos encontrados na parede celular de fungos, amplamente estudados em função de seu valor medicinal (Synytsya e Novak, 2013). Entre as atividades farmacológicas relatadas para os $\beta$-glucanos estão antimicrobiana (Wisbeck et al., 2002; Iwalokun et al., 2007), antinociceptiva (Iwalokun et al., 2007; Smiderle et al., 2008), antitumoral (Dalonso et al., 2010), antioxidante e antihepatotóxico (Ajith e Janardhanan, 2002) e próimunomodulatória (Bao et al., 2001).

Colleoni-Sirghie et al. (2003) relatam que a aplicação terapêutica destes polissacarídeos parece depender da estrutura química e da conformação espacial de cada macromolécula, sendo que pequenas diferenças estruturais de cada polímero resultam em características peculiares para novas aplicações.

Portanto, informações sobre isolamento, estrutura química, propriedades físico-químicas e 
bioatividades sobre estes polímeros são essenciais para pesquisas futuras e aplicação destes polissacarídeos (Zhang et al., 2003).

Assim, este trabalho teve como objetivo extrair, fracionar, purificar e analisar a composição monossacarídica dos polissacarídeos presentes na biomassa micelial de Pleurotus sajor-caju, usando água quente e fria como solvente.

\section{METODOLOGIA}

Pleurotus sajor-caju CCB 019 foi obtido do Centro de Cultivo de Basidiomicetos da Universidade de São Paulo. A linhagem foi mantida em meio sólido TDA (Trigo Dextrose Ágar) (Furlan et al., 1997), sob refrigeração $\left(4^{\circ} \mathrm{C}\right)$ e os repiques feitos a cada três meses.

\subsection{Produção de biomassa por cultivo submerso}

O cultivo submerso foi realizado em biorreator de mistura completa, com volume de trabalho de 4L, utilizando meio POL modificado (Assis et al., 2013), com concentrações iniciais de glicose e $\mathrm{CaCO}_{3}$ de 20 e $1 \mathrm{~g} / \mathrm{L}$, respectivamente. $\mathrm{O} \mathrm{pH}$ foi fixado em 4,0 e a temperatura em $30^{\circ} \mathrm{C}$. O fluxo de ar foi mantido em $0,4 \mathrm{vvm}$ (volume de ar por volume de meio por minuto) e a agitação em $350 \mathrm{~min}^{-1}$, resultando no valor de $\mathrm{K}_{\mathrm{L}}$ a (coeficiente volumétrico de transferência de oxigênio) igual a $15 \mathrm{~h}^{-1}$ no início do cultivo. O cultivo foi finalizado após a verificação do consumo total da glicose, através de método enzimático. Após, o caldo de cultivo e a biomassa, obtidos do cultivo submerso, foram separados por filtração à vácuo.

\subsection{Processos de extração e fracionamento dos polissacarídeos da biomassa}

A biomassa foi liofilizada e o conteúdo lipídico foi extraído por refluxo em aparelho Soxhlet utilizando-se como solvente uma mistura de clorofórmio: metanol $(2: 1$, v/v) por 24 horas. O micélio proveniente da deslipidificação (resíduo I) foi solubilizado em água destilada e mantido sob agitação a $10^{\circ} \mathrm{C}$ por 24 horas. Após, o extrato aquoso frio foi recuperado por centrifugação e ao resíduo foi adicionado água, sendo o processo de extração repetido por 10 vezes. Os extratos provenientes dos 10 processos foram juntados, concentrados em rotaevaporador à vácuo e liofilizados, obtendo-se o extrato aquoso frio $(\mathrm{CW})$. Os procedimentos de fracionamento e purificação do extrato aquoso frio estão apresentados na Figura 1. 


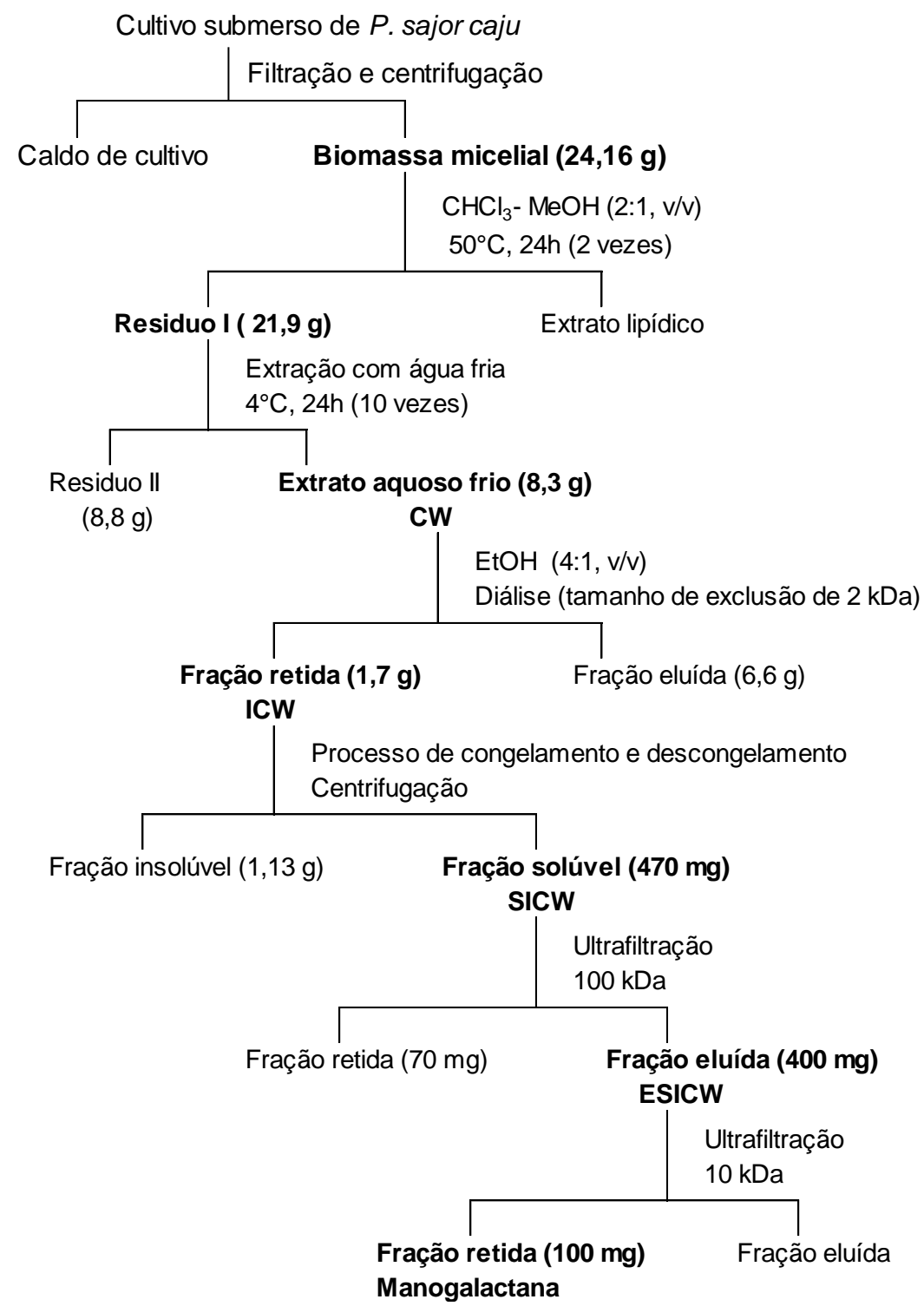

Figura 1 - Fluxograma de extração aquosa a frio de biomassa micelial de $P$. sajor-caju.

O resíduo da extração aquosa a frio (resíduo II) foi novamente solubilizado em água destilada a $100^{\circ} \mathrm{C}$ sob agitação por 24 horas (sob refluxo). $\mathrm{O}$ extrato aquoso quente foi separado do resíduo por centrifugação, e novo processo de extração com água quente foi realizado com o resíduo por 10 vezes. Os extratos obtidos foram reunidos, concentrados e liofilizados obtendo-se então o extrato aquoso quente (HW). Os procedimentos de fracionamento do extrato aquoso quente estão apresentados na Figura 2. 


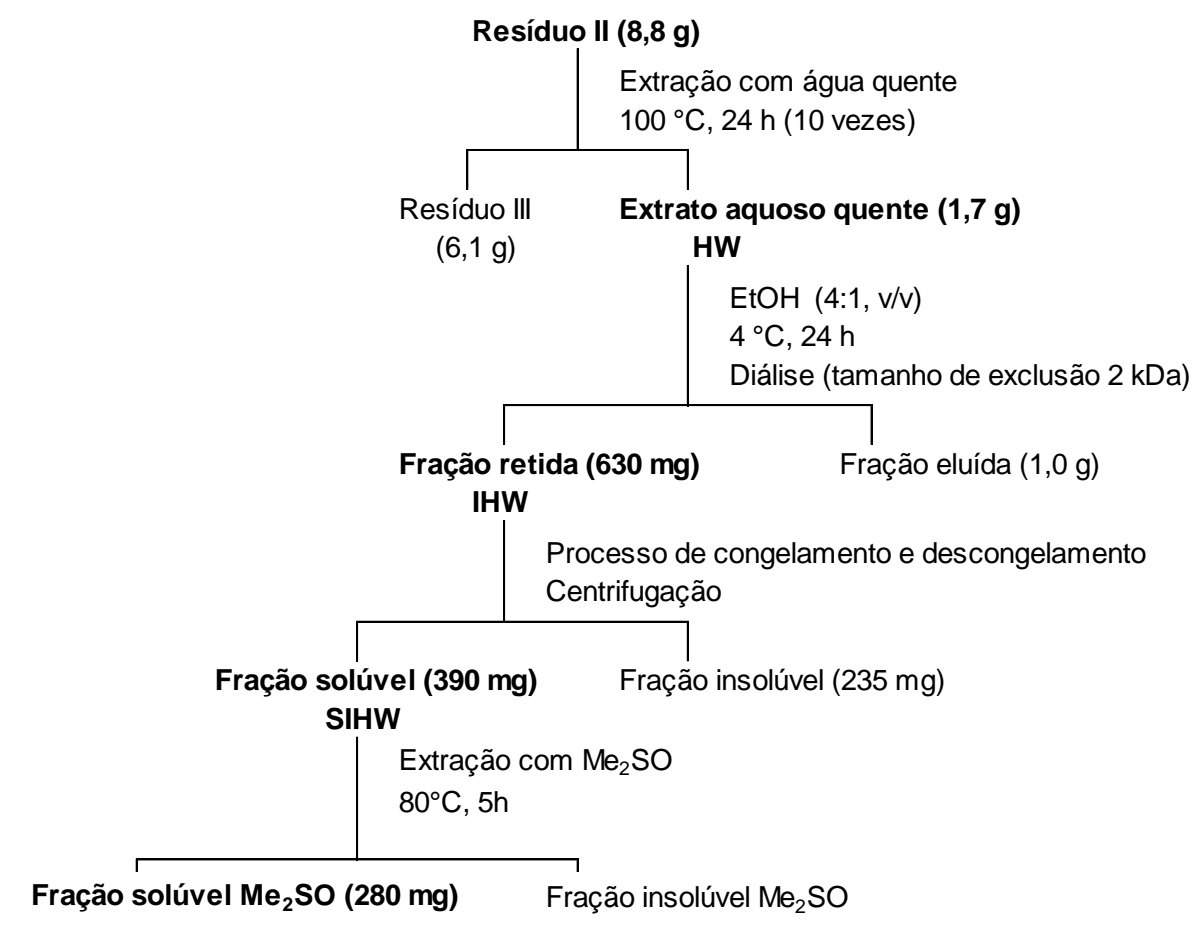

Figura 2 - Fluxograma de extração aquosa a quente a partir do resíduo da extração aquosa a frio de biomassa micelial de $P$. sajor-caju.

\subsection{Análises de composição monossacarídica e homogeneidade dos polissacarídeos}

A cada etapa de fracionamento, tanto do extrato aquoso frio quanto do extrato aquoso quente, foram realizadas análises de composição monossacarídica. Após o processo de congelamento e descongelamento, as amostras obtidas a partir da fração solúvel foram analisadas quanto a sua homogeneidade.

Para composição monossacarídica, amostras de $2 \mathrm{mg}$ foram colocadas em tubo de ensaio, com adição de $1 \mathrm{~mL}$ de TFA $1 \mathrm{M}$ e deixadas a $100^{\circ} \mathrm{C}$ por $12 \mathrm{~h}$ (Gorin et al., 1996). O excesso de ácido foi evaporado, foi adicionado $1 \mathrm{~mL}$ de água e boridreto de sódio até $\mathrm{pH}$ 9-10, sendo deixado reagir por $24 \mathrm{~h}$ à temperatura ambiente, para ocorrer a redução. Após, foi adicionado ácido acético até $\mathrm{pH}$ 3-4. O conteúdo foi vertido em balão e evaporado a vácuo (Fisatom) à $40^{\circ} \mathrm{C}$ até secura. $\mathrm{O}$ conteúdo do balão foi lavado 3 vezes com metanol. Após, foi adicionado $1 \mathrm{~mL}$ de metanol, o conteúdo vertido em tubo e este deixado aberto para evaporação do excesso de metanol. Após, foi adicionado $300 \mu \mathrm{L}$ de piridina e $300 \mu \mathrm{L}$ de anidrido acético e deixado por $12 \mathrm{~h}$ para ocorrer a acetilação (Wolfrom e Thompson, 1963b, Wolfrom e Thompson, 1963a). Ao tubo foi adicionado $1 \mathrm{~mL}$ de clorofórmio e a fase clorofórmica foi lavada com sulfato de cobre $5 \%$. Em seguida, a solução foi filtrada e os alditóis acetilados foram analisados em um cromatógrafo a gás (Varian Saturn 2000R - 3800) acoplado a um espectrômetro de massa (Varian Ion-Trap 
2000R), utilizando uma coluna capilar de sílica fundida DB-225 (30 m x 0,25 mm) e hélio ultrapuro, a um fluxo de $1 \mathrm{~mL} / \mathrm{min}$, como gás de arraste. Os acetatos de alditóis foram analisados em rampa de temperatura de 50 a $220^{\circ} \mathrm{C}\left(40^{\circ} \mathrm{C} / \mathrm{min}\right)$.

As análises de homogeneidade foram realizadas no aparelho da Wyatt Technology, equipado com um Cromatógrafo de Exclusão Estérica de Alto Desempenho (HPSEC) com um detector de índice de refração, modelo Waters 2410, e um detector de espalhamento de laser multiângulo (MALLS) a 632,8 nm, modelo Dawn DSP, que promove a leitura do espalhamento de luz, que é captado em diferentes intensidades por detectores em diferentes ângulos. Como eluente foi utilizada uma solução de $\mathrm{NaNO}_{2}$ a 0,1 M. As amostras foram solubilizadas no eluente em concentração final de $1 \mathrm{mg} / \mathrm{mL}$ e filtradas em membranas com diâmetro médio de poro de $0,22 \mu \mathrm{m}$.

\section{RESULTADOS E DISCUSSÕES}

\subsection{Extrato aquoso frio}

O micélio deslipidificado foi colocado em água e mantido em agitação para obtenção do extrato aquoso frio. Etanol foi adicionado a esse extrato e, após 24 horas, o precitado formado foi separado por centrifugação, dialisado, e foi submetido ao processo de congelamento/descongelamento.

A fração solúvel no gelo/degelo (SICW) apresentou composição monossacarídica formada por 3,3\% de Rha, 3,6\% de Ara, 2,0\% de Xyl, 23,1\% de Gal, 21,8\% de Me-Gal, 37,4\% de Man e 8,8\% de Glu, com perfil de eluição apresentado na Figura 3.

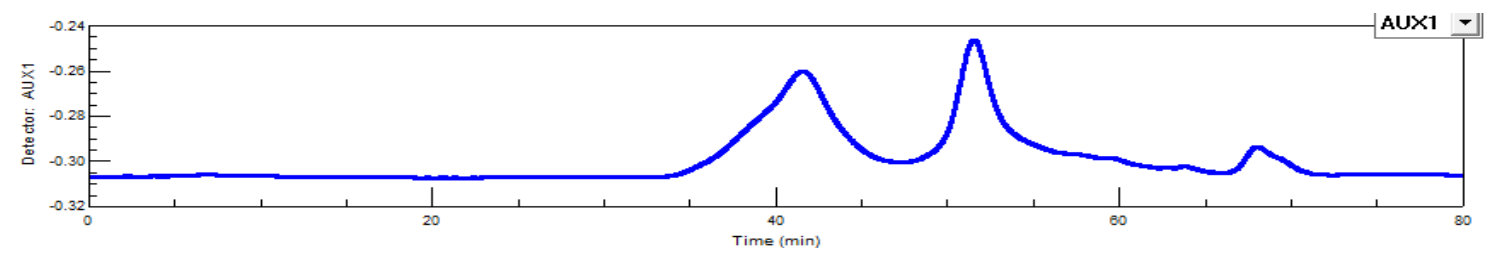

Figura 3 - Perfil de eluição da fração SICW.

O perfil observado (Figura 3) demonstra uma solução com várias moléculas. Então, SICW foi submetida a ultrafiltração em membrana de 100kDa. A fração eluída (filtrado) na membrana de $100 \mathrm{kDa}(\mathrm{ESICW})$ apresentou composição monossacarídica formada por $13,1 \%$ de Me-Gal, 18,1\% de Gal, 24,1\% de Man e 44,7\% de Glc e perfil de eluição apresentado na Figura 4. 


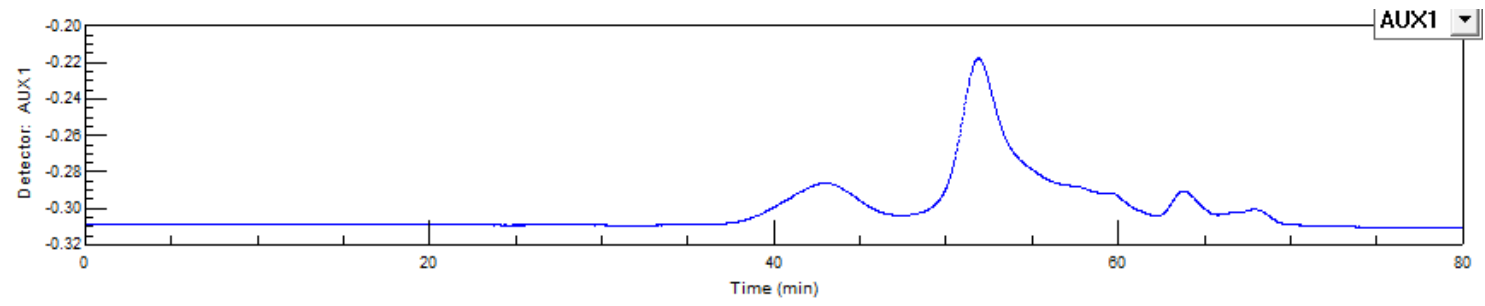

Figura 4 - Perfil de eluição da fração ESICW.

O processo de ultrafiltração em membrana de $100 \mathrm{kDa}$ foi efetivo, pois promoveu a separação dos dois picos observado na Figura 02. Porém, não foi eficiente pois ainda há presença de um segundo pico (menor intensidade) na fração eluída da membrana de $100 \mathrm{kDa}$. Então, a fração eluída foi submetida novamente a ultrafiltração em membrana de $100 \mathrm{kDa}$ e, em seguida, em membrana de ultrafiltração de $10 \mathrm{kDa}$, para retirar picos próximos a 60 minutos.

A composição monossacarídica da fração retida em membrana de $10 \mathrm{kDa}$ e permeada (eluída) em $100 \mathrm{kDa}$ (manogalactana) foi 15,8\% de Glc, 22,3\% de Me-Gal, 28,1\% de Gal e $33,8 \%$ de Man e o perfil de eluição está apresentado na Figura 5.

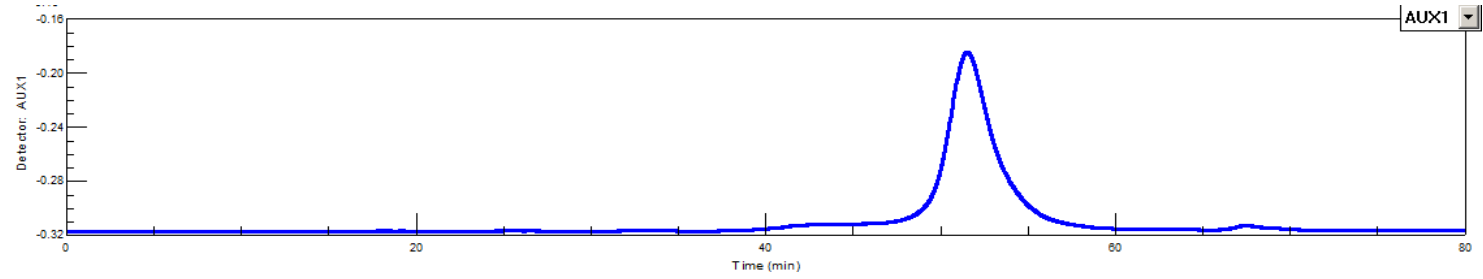

Figura 5 - Perfil de eluição da manogalactana.

A Figura 5 demonstra um perfil homogêneo para a fração eluída em membrana de ultrafiltração em $100 \mathrm{kDa}$ e retida em $10 \mathrm{kDa}$, indicando a presença de um polissacarídeo, uma manogalactana.

Manogalactanas similares foram isoladas de extrato aquoso frio de corpos frutíferos de $P$. pulmonarius (Smiderle et al., 2008), de extrato aquoso quente de P. ostreatus (Sun e Liu, 2009), assim como, de extrato aquoso quente de exopolissacarídeos de $P$. ostreatoroseus (Rosado et al., 2002).

\subsection{Extrato aquoso quente}

O resíduo de micélio da extração aquosa a frio foi solubilizado em água e mantido em agitação e aquecimento para obtenção do extrato aquoso quente. Etanol foi adicionado a esse extrato, após 24 horas, o precitado formado foi separado por centrifugação, dialisado, e foi submetido ao processo de congelamento/descongelamento. 
A fração solúvel em gelo/degelo (SIHW) apresentou composição monossacarídica: 4,6\% de Xyl, 28,8\% de Man e 66,6\% de Glc, com perfil de eluição apresentado na Figura 6.

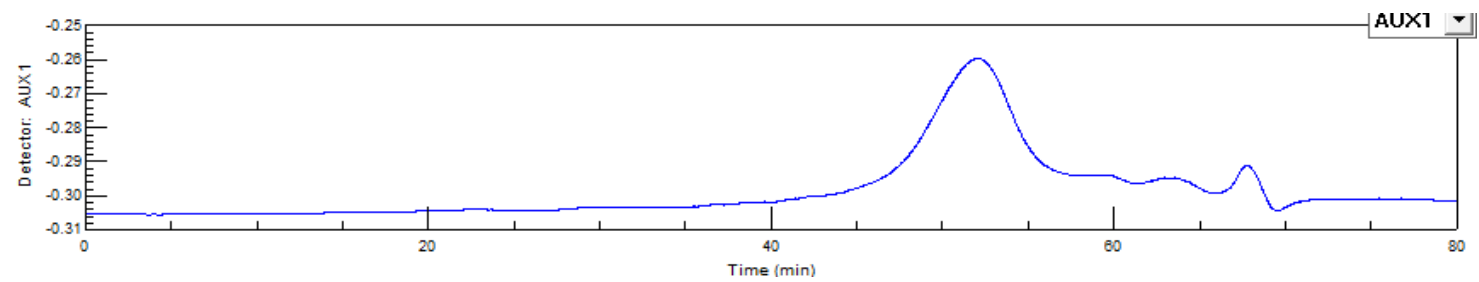

Figura 6 - Perfil de eluição da fração SIHW.

A SIHW foi solubilizada em dimetilsulfóxido a quente e dialisada em membrana de 6-8 kDa. A fração solúvel retida apresentou composição monossacarídica de $28,6 \%$ de Man e 71,4\% de Glc. O perfil de eluição (Figura 7) desta fração foi heterogêneo, com picos pequenos próximos a região de 60 minutos.

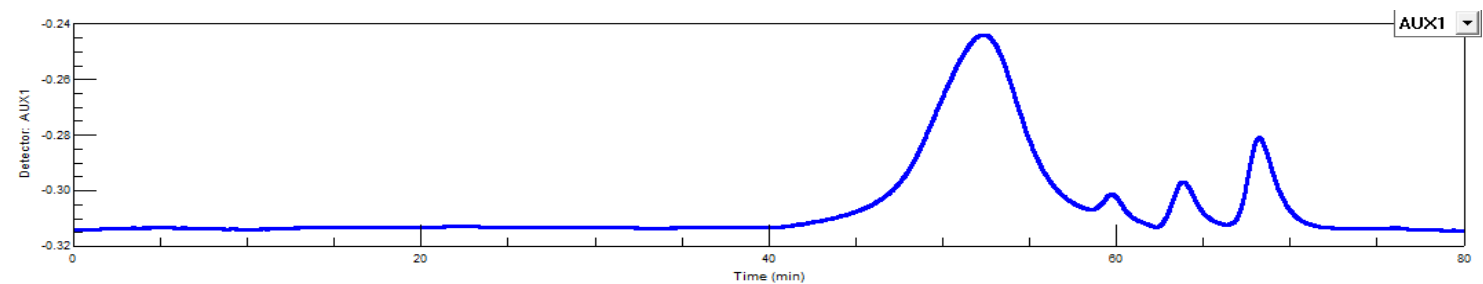

Figura 7 - Perfil de eluição da fração solúvel em $\mathrm{Me}_{2} \mathrm{SO}$.

Zhang et al. (2007) identificaram manoglucanas obtidas de corpos frutíferos de Lentinus edodes e de Ganoderma lucidum.

\section{CONCLUSÃO}

Neste trabalho foi isolada uma manogalactana da biomassa micelial de $P$. sajor-caju através de extração aquosa a frio e uma manoglucana do extrato aquoso quente. A presença destas estruturas já foi relatada em outros trabalhos, isoladas de corpos de frutificação de cogumelos comestíveis.

\section{AGRADECIMENTOS}

Os autores agradecem ao Centro de Cultivo de Basideomicetos (CCB), ao CNPq e FAP-UNIVILLE.

\section{REFERENCIAS}

AJITH, T.A.; JANARDHANAN, K.K. Antioxidant and antihepatotoxic activities of Phellinus rimosus (Berk) Pilat. J. Ethnopharmacol., v. 81, n. 3, p. 387-391, 2002.

ASSIS, I.S.; CHAVES, M.B.; SILVEIRA, M.L.L.; GERN, R.M.M.; WISBECK, E.; FURIGO JÚNIOR, A.; FURLAN, S.A. Production of bioactive compounds with antitumor activity against Sarcoma 180 by Pleurotus sajor-caju. J. Medic. Food, v. 16, n. 11, p. 1004-1012, 2013. 


\section{9 a 22 de outubro de 2014 \\ Florianópolis/SC}

BAO, X.; DUAN J.; FANG X.; FANG, J. Chemical modifications of the $(1 \rightarrow 3)$ - $\alpha$-D-glucan from spores of Ganoderma lucidum and investigation of their physicochemical properties and immunological activity. Carbohyd. Res., v. 336, n. 2, p. 127-140, 2001.

BONATTI, M.; KARNOPP, P.; SOARES, H.M.; FURLAN, S.A. Evaluation of Pleurotus ostreatus and Pleurotus sajor-caju nutritional characteristics when cultivated in different lignocellulosic wastes. Food Chem., v. 88, n. 3, p. 425-428, 2004.

COLLEONI-SIRGHIE, M.; FULTON, D.B.; WHITE, P.J. Structural features of water soluble $(1,3)(1,4)$ $\beta$-D-glucans from high- $\beta$-glucan and tradicional oat lines. Carbohydr. Polym., v. 54, n. 2, p. $237-$ 249, 2003.

DALONSO, N.; SOUZA, R.; SILVEIRA, M.L.L.; RUZZA, A.A.; WAGNER, T.M.; WISBECK, E.; FURLAN, S.A. Characterization and antineoplasic effect of extracts obtained from Pleurotus sajorcaju fruiting bodies. Appl. Biochem. Biotech., v. 160, n. 8, p. 2265- 2274, 2010.

FURLAN, S.A.; VIRMOND, L.J.; MIERS, D.; BONATTI, M.; GERN, R.M.M.; JONAS,R. Mushroom strains able to grow at high temperatures and low pH values. World J. Microb. Biot., v. 13, p. 689692, 1997.

GORIN, P.A.J.; TEIXEIRA, A.Z.A.; TRAVASSOS, L.R.; LABOURIAU, M.L.S.; IACOMINI, M. Characterization of carbohydrate components of an unusual hydrogel formed by seed coats of Magonia pubescens (Tingui). Carbohyd. Res., v. 282, n. 2, p. 325-333, 1996.

IWALOKUN, B.A.; USEN, U.A.; OTUNBA, A.A.; OLUKOYA, D.K. Comparative phytochemical evoluation, antimicrobial and antioxidant properties of Pleurotus ostreatus. African Journal of Biotechnology, v. 6, n. 15, p. 1732-1739, 2007.

MANZI, P.; PIZZOFERATO, L. Beta-glucans in edible mushrooms. Food Chem., v. 68, n. 3, p. 315-318, 2000.

RAMPINELLI, J.R; SILVEIRA, M.L.L.; GERN, R.M.M.; FURLAN, S.A.; Ninow, J.L.; WISBECK, E. Valor nutricional de Pleurotus djamor cultivado em palha de bananeira. Alimentos e Nutrição (UNESP. Marilia), v. 21, p. 195-200, 2010.

ROSADO, F.R.; CARBONERO, E.; KEMMELMEIER, C.; TISCHER, C.A.; GORIN, P.A.J.; IACOMINI, M. A partially 3-O-methylated $(1 \rightarrow 4)$-linked $\alpha$-D-galactan and $\alpha$-D-mannan from Pleurotus ostreatoroseus Sing. FEMS Microbiol. Lett., v. 212, p. 261-265, 2002.

SMIDERLE, F.R.; OLSEN, L.M.; CARBONERO, E.R.; MARCON, R.; BAGGIO, C.H.; FREITAS, C.S.; SANTOS, A.R.S.; TORRI, G.; GORIN, P.A.J.; IACOMINI, M. A 3-O-methylated mannogalactan from Pleurotus pulmonarius: Structure and antinociceptive effect. Phytochem, v. 69, p. 2731-2736, 2008

SUN, Y.; LIU, J. Purification, structure and immunobiological activity of a water-soluble polysaccharide from the fruiting body of Pleurotus ostreatus. Bioresource Technol., v. 100, p. 983-986, 2009.

SYNYTSYA A.; NOVÁK, M. Structural diversity of fungal glucans. Carbohydr. Polym., v. 92, n. 1, p. 792-809, 2013.

WISBECK, E.; ROBERT, A.P.; FURLAN, S.A. Avaliação da produção de agentes antimicrobianos por fungos do gênero Pleurotus. Revista Saúde e Ambiente (UNIVILLE), v. 3, p. 7-10, 2002.

WOLFROM, M.L.; THOMPSON, A. Reduction with sodium borohydride. Methods in Carbohydrate Chemistry, v. 2, p. 65-67, 1963 a.

WOLFROM, M.L.; THOMPSON, A. Acetylation. Methods in Carbohydrate Chemistry, v. 2, p. 211-215, $1963 b$. 
ZHANG, M.; ZHANG, L.; CHEUNG, P.C. Molecular mass and chain conformation of carboxymethylate derivatives of beta-glucan from sclerotia of Pleurotus tuber-regium. Biopolymers. v. 68, n.2, p.150$159,2003$.

ZHANG, M.; CUI, S.W.; CHEUNG, P.C.K.; WANG, Q. Antitumor polysaccharides from mushrooms: a review on their isolation process, structural characteristics and antitumor activity. Trends in Food Sci. Tech., v.18, n.1, p.4-19, 2007. 